\title{
Contribution à l'étude des Microphallidae Travassos, 1920 (Trematoda).
}

XXXIV. Redescription de Levinseniella bowensis Johnston, 1917

\author{
par J.-C. PEARSON * et S. DEBLOCK ** \\ * Department of Parasitology, University of Queensland, St Lucia, Brisbane Australia, 4067. \\ ** Laboratoire de Parasitologie, Faculté de Pharmacie, rue du Pr-Laguesse, F 59045 Lille.
}

\section{Résumé:}

D'après les types de l'espèce, L. howensis se classe dans le sous-genre Levinseniella et non Monarrhenos comme il était supposé jusqu'à présent. L'atrium génital de l'espèce comporte deux poches mâles et une poche femelle.

\section{Summary:}

Contribution to the study of Microphallitae Travassos, 1920 (Trematoda). XXXIV. Redescription of Levinseniella hovensis Johnston, 1917.

According to the type of L. howensis the species belongs to the subgenus Levinseniella instead of the subgenus Monarrhenos as believed before. The genital atrium has iwo male pockets and one female pouch.

L'inventaire des collections d'helminthes de S. J. Johnston a mis à jour des exemplaires de Levinseniella howensis Johnston, 1917. Leur observation nécessite une redescription destinée à corriger la définition de l'espèce.

Accepté le 18 décembre 1978. 


\section{Levinseniella bowensis Johnston, 1917.}

Hôte : Charadrius dominicus Müll.

HaBitat : gros intestin.

Localisation GÉOgRaphiQue: Lord Howe Island (Australie).

Matériel obSERvé: $1^{\circ}$ Une préparation de S. J. Johnston comportant 4 exemplaires montés au baume, décolorés mais bien observables; ils représentent vraisemblablement les syntypes de l'espèce. $2^{\circ}$ Trois préparations de T. H. Johnston comportant quarante exemplaires colorés à l'hémalun, du même hôte et de même provenance géographique que les précédents; ils paraissent constituer soit une nouvelle récolte des parasites, soit un reliquat des récoltes du premier auteur monté ultérieurement. Il s'agit bien de la même espèce. Nos mensurations résultent de l'observation de 10 exemplaires (les moyennes sont citées en premier lieu, suivies des tailles extrêmes entre parenthèses).

\section{Description}

L'anatomie des parasites est conforme à la définition du genre, avec les particularités ci-après : Corps long de $926 \mu \mathrm{m}$ (extrêmes : 720 et $1100 \mu \mathrm{m}) \times 360 \mu \mathrm{m}$ de largeur maximale (extrêmes : 320 et $400 \mu \mathrm{m}$ ). Epines tégumentaires de $6 \mu \mathrm{m}$ au niveau du pharynx, avec une densité de 36 épines pour $625 \mu \mathrm{m}^{2}(25 \times 25 \mu \mathrm{m})$. Ventouse orale de $70 \mu \mathrm{m}$ de diamètre (60 et 80 ), arrondie régulièrement, dépourvue d'auricule et de membrane basale épaissie ; le sphincter pré-pharyngien est présent mais discret. Prépharynx de $90 \mu \mathrm{m}$ (30 et 130). Pharynx de $56 \times 48 \mu \mathrm{m} \quad(50-63 \times 42-53)$. Esophage de $115 \mu \mathrm{m}(60$ et 140$) \times 13 \mu \mathrm{m}$ de diamètre. Coeca de $380 \mu \mathrm{m}(340-440)$ et de 30-40 $\mu \mathrm{m}$ de diamètre ; leur fond atteint le niveau du plan équatorial de l'acétabulum. Ventouse ventrale arrondie située à $206 \mu \mathrm{m}$ du bord postérieur du corps et mesurant $75 \times 67 \mu \mathrm{m}(65-80 \times 65-75 \mu \mathrm{m})$; elle est entourée des fibres musculaires acétabulo-atriales propres au genre Levinseniella, mais assez discrètement figurées. Rapport V.O./V.V. $=1$. Testicules de $55 \times 100 \mu \mathrm{m}$. Vésicule séminale de $90 \times 50 \mu \mathrm{m}$. Pars prostatica de $75 \times 25 \mu \mathrm{m}$. Ovaire de $55 \times 95 \mu \mathrm{m}$. Eufs régulièrement ovalaires mesurant selon les distomes $16-22 \times 10-13 \mu \mathrm{m}$; la plupart d'entre eux présentent un épaississement annulaire disposé à distance de chacun des deux pôles à la façon des cercles d'un tonneau. La moitié antérieure du système excréteur est bien visible sur certain exemplaires (canaux et solénocytes), et 14 des 16 solénocytes ont été localisés; un des solénocytes de la deuxième paire antérieure se situe de façon constante dans l'angle interne des coecums. Le reste de l'anatomie et des organes comme dans les autres espèces du genre, à l'exception du sinus génital. 
Fig. 1. Levinseniella (L.) howensis Johnston, 1917. Type ou syntype. Vue ventrale.

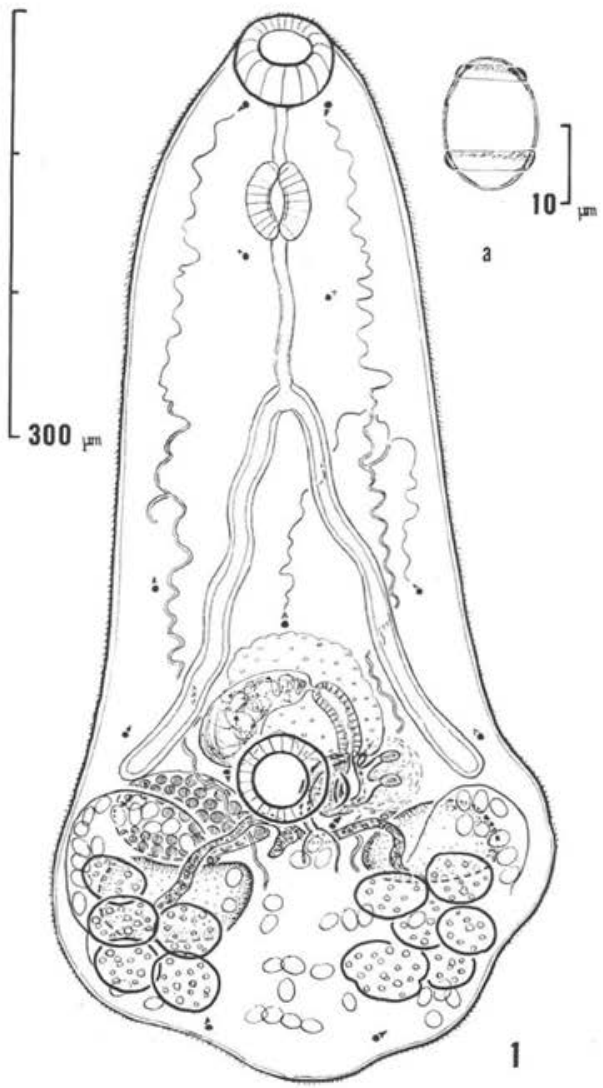
a) Euf.

SINUS GÉNITAL : L'atrium génital s'organise comme chez les Levinseniella (Levinseniella). Sous le pore génital en forme de fente longitudinale, l'atrium est occupé par la papille mâle massuée de $20-25 \times 10-15 \mu \mathrm{m}$, habituellement assez mal visible. Du côté gauche, l'atrium émet successivement et dans un même plan deux diverticules (ou poches mâles) sensiblement parallèles l'un à l'autre et sub-égaux, le premier long de 20-30 et le second de 15-25 $\mu \mathrm{m}$, prolongés chacun d'une masse charnue ovoïde, creuse, de 15-20 × 11-13 $\mu \mathrm{m}$ de diamètre, et inerme intérieurement. Le massif parenchymateux qui les entoure mesure $80 \times 55 \mu \mathrm{m}(45-70 \times 70-100 \mu \mathrm{m})$. Du côté droit, l'atrium émet un diverticule assez vaste de $50 \times 34 \mu \mathrm{m}(46-56 \times 26-40 \mu \mathrm{m})$, ou poche femelle dont la position varie selon les exemplaires: partiellement engagé en dessous de l'hémiacétabulum gauche, tantôt du côté antérieur tantôt du côté postérieur; ses parois ne sont pas sclérotinisées, mais seulement épaissies et très colorables. Le fond de l'atrium situé entre la racine de la papille mâle et le diverticule mâle antérieur reçoit l'ouverture du métraterme. 

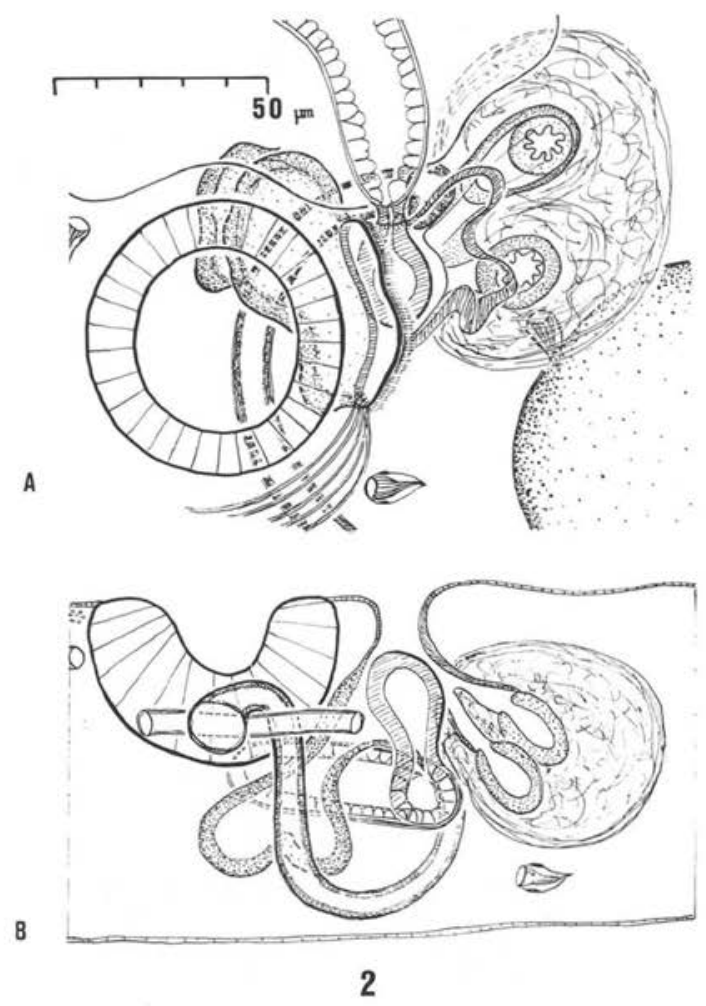

Fig. 2. L. howensis. Détails de l'atrium génital. A: vue ventrale. B : reconstitution semi-schématique de l'atrium génital projeté sur une coupe transversale passant par le pore génital et la ventouse ventrale. De droite à gauche: les deux poches mâles et un solénocyte dorsal; l'ouverture métratermique dans un plan postérieur; la papille mâle dressée et le pore génital; la pars prostatica; la poche femelle, la ventouse ventrale et le franchissement ventral du vitelloducte transverse par l'utérus.

\section{Discussion}

L'espèce se caractérise par une assez grande taille relative, une petite ventouse orale, un atrium à deux poches mâles et une poche femelle, ainsi que par une ornementation spéciale de l'enveloppe des œufs.

De par l'anatomie de son atrium génital, l'espèce howensis fera désormais partie du sous-genre Levinseniella, et non du sous-genre Monarrhenos comme il avait été primitivement supposé en absence de mention de la poche atriale femelle (Deblock et Pearson, 1970; Deblock, 1971). L'usage de la clé de détermination des espèces du genre Levinseniella de ces auteurs conduit par les reports 1,3 et 4 à classer l'espèce howensis au voisinage de deux espèces nouvellement décrites, pourvues d'un atrium génital de même type général: L. cipangi Deblock et Pearson, 1970 et $L$. heardi Canaris, 1971. Les trois espèces sont morphologiquement très semblables et les différences de mensuration apparaissent peu significatives; toutefois, l'ornementation de la coque des œufs de howensis paraît devoir suffire à individualiser les deux autres espèces 
de celle-ci. A notre connaissance, aucun autre Microphallidé n'en présente de semblable. $L$. heardi n'a été identifié à l'heure actuelle que sur les rivages de l'Océan Indien (Kénya) chez un reptile se nourrissant de crustacés de rivages. Le cas de $L$. ( $L$.) cipangi est illustré par les figures 3 et 4 . Les deux poches mâles sont situées chacune à l'extrêmité des branches d'un $\mathrm{Y}$, et non à celle de deux branches semi-parallèles comme chez howensis. Par ailleurs, le solénocyte de la seconde paire antérieure n'est pas intercoecal. Le parasite n'a été identifié à l'heure actuelle que chez un oiseau de rivage de la Mer du Japon.
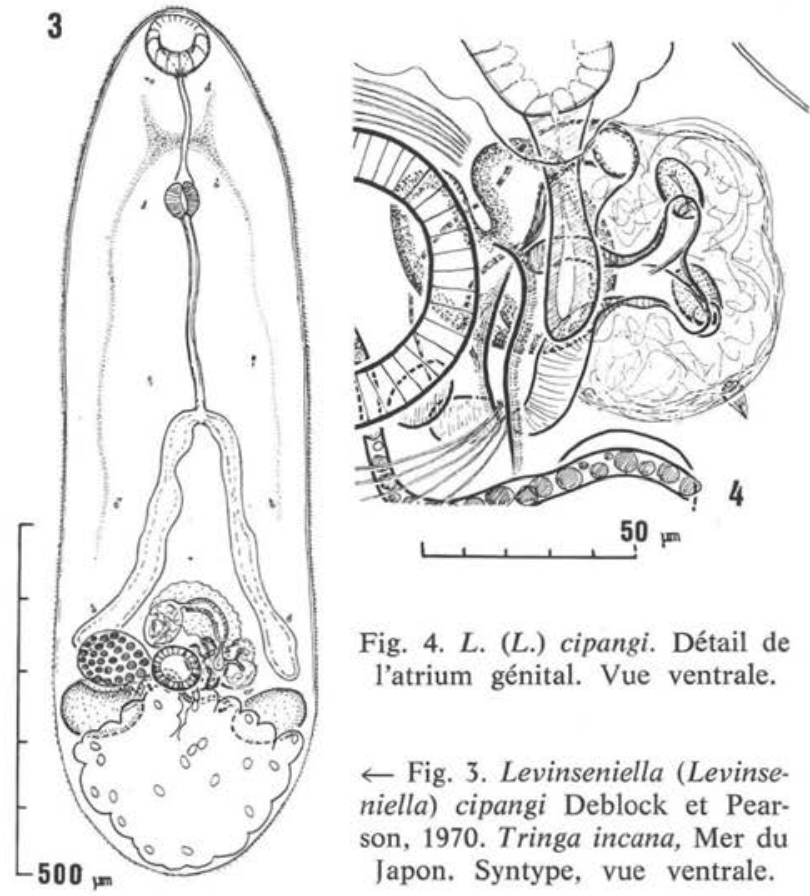

Fig. 4. L. (L.) cipangi. Détail de l'atrium génital. Vue ventrale.

$\leftarrow$ Fig. 3. Levinseniella (Levinseniella) cipangi Deblock et Pearson, 1970. Tringa incana, Mer du Japon. Syntype, vue ventrale.

Les espèces signalées jusqu'à présent sur les rivages ouest du Pacifique sont les suivantes :

I - GenRe Levinseniella (Monarrhenos) :

- Une poche mâle unique :

L. (M.) monodactyla Deblock et Pearson, 1970 (Australie, Japon) ; syn. : L. conicostoma Bridgman et coll., 1972. 
II - GENRE Levinseniella (Levinseniella) :

a) Une poche mâle unique :

L. (L.) bucephalae (Yamaguti, 1935) Yamaguti, 1939 (Japon, Sibérie orientale) ; syn. : L. acanthophalla Oschmarin, 1963 ; L. bucephalae tringae Oschmarin, 1963 ; Oschmarinotrema acanthophallus (Oschm.) Yamag., 1971.

b) Deux poches mâles :

L. (L.) howensis Jonhston, 1917 (Australie).

L. (L.) cipangi Deblock et Pearson, 1970 (Sibérie orientale) ; syn. : L. bucephalae sensu Belopolskaia, 1954.

L. (L.) cryptacetabula Oschmarin et coll., 1970 (Viêt-nam).

L. (L.) mayna Oschmarin et Demshin, 1972 (Viêt-nam).

c) Trois poches mâles :

L. (L.) ryjikovi Beloposkaia, 1963 (Sibérie orientale).

d) Quatre poches mâles :

L. (L.) miyazakii (Yamaguti, 1975) Deblock, 1978 (Japon) ; syn. : Megalophallus m. Yamag. ; ? L. bucephalae sensu Ryjikof et Timofeeva, 1962.

e) Quatre ou cinq poches mâles :

L. (L.) microovata Belopolskaia, 1958 (Sibérie orientale, Australie).

f) Nombre de poches mâles indéterminé :

L. (L.) kamtshatica Morosov, 1960 (Kamtchatka) et Levinseniella occultacetabulum (Oschmarin, 1963).

Le statut de quelques-unes de ces espèces ne paraît pas être établi avec toute la précision souhaitable. En outre, il existe en Alaska et au Canada un Levinseniella (Lev.) tantôt à trois, tantôt à quatre poches mâles que nous n'avons pas su distinguer de l'espèce propinqua européenne; il paraît fort possible, et même très vraisemblable, que cette forme s'étende aussi sur les rives asiatiques du Pacifique. Ses rapports avec les espèces citées en référence ci-dessus ne sont pas établis.

\section{Bibliographie}

La liste ci-dessous complète les références citées par Yamaguti, 1971 ou par Deblock, 1971.

Bridgman (J.F.), Otagaki (K.), Shitanda (1) et Tada (K.), 1972: Nine metacercariae (Microphallidae : Trematoda) from arthropods of the Japan Inland Sea, Kagawa Prefecture, including the description of Levinseniella conicostoma n. sp. Shikoku Christian College Treatises, 23, 35-61.

Canaris (A.G.), 1971 : Some Microphallids collected in Kenya. Parasitol., 62, 53-61.

Deblock (S.), 1971: Contribution à l'étude des Microphallidae Travassos, 1920. XXIV. Tentative de phylogénie et de taxonomie. Bull. Mus. Nat. Hist. Nat., $3^{e}$ ser. (7), Zool., 7, 353-468.

Deblock (S.), 1978: Invalidation du genre Austromicrophallus Szidat, 1964 (Trematoda: Microphallidae). Ann. Parasitol. Hum. Comp., 53, 47-52. 
Deblock (S.) et Pearson (J.C.), 1970: Contribution à l'étude des Microphallidae Travassos, 1920 (Trematoda). XXII. De deux Levinseniella d'Australie dont un nouveau: Lev. (Monarrhenos) monodactyla. Essai de clé diagnostique des espèces du genre. Ann. Parasitol. Hum. Comp., 45, 773-791.

Johnston (S.J.), 1917 : On the trematodes of Australian birds. Jl Proc. R. Soc. N. S. Wales, 50, 187261.

Oshmarin (P.G.), 1970: Trématodes des animaux domestiques et sauvages de la République Démocratique du Viêt-nam, in Oshmarin (P.), Mamaev (L.) et Lebedev (B.): Helminthes des animaux du Sud-Est asiatique. Publ. Acad. Sci. U.R.S.S., 5-126.

Oshmarin (P.G.) et Demshin (N.), 1972: Helminthes d'animaux domestiques et sauvages de la République démocratique du Viêt-nam. Rapports Inst. Biol. et Pédologique d'Extr. Or., Publ. Acad. Sci. U.R.S.S. 11 (114), 5-115 (Microphallidae p. 17-18).

Yamaguti (S.), 1971: Synopsis of digenetic trematodes of Vertebrates. I et II. Keigaku Publ. $C^{\circ}$, Tokyo, Japon, 1074 p. et 349 pl.

Yamaguti (S.), 1975: A synoptical review of life histories of digenetic trematodes of Vertebrates which special reference to the morphology of larval forms. Keigaku Publ. $C^{\circ}$, Tokyo, Japon, 590 p. 